\title{
Disease Surveillance and Achieving Synergy In Public Health Quality Improvement
}

\author{
Peggy A. Honoré ${ }^{\star 1}$ and Laura C. Streichert ${ }^{2}$ \\ 1U.S. Department of Health and Human Services, Washington, DC, USA; ${ }^{2}$ International Society for Disease Surveillance, Brighton, \\ MA, USA
}

\section{Objective}

To examine disease surveillance in the context of a new national framework for public health quality and to solicit input from practitioners, researchers, and other stakeholders to identify potential metrics, pivotal research questions, and actions for achieving synergy between surveillance practice and public health quality.

\section{Introduction}

National efforts to improve quality in public health are closely tied to advancing capabilities in disease surveillance. Measures of public health quality provide data to demonstrate how public health programs, services, policies, and research achieve desired health outcomes and impact population health. They also reveal opportunities for innovations and improvements. Similar quality improvement efforts in the health care system are beginning to bear fruit. There has been a need, however, for a framework for assessing public health quality that provides a standard, yet is flexible and relevant to agencies at all levels.

The U.S. Health and Human Services (HHS) Office of the Assistant Secretary for Health, working with stakeholders, recently developed and released a Consensus Statement on Quality in the Public Health System that introduces a novel evaluation framework. They identified nine aims that are fundamental to public health quality improvement efforts and six cross-cutting priority areas for improvement, including population health metrics and information technology; workforce development; and evidence-based practices (1).

Applying the HHS framework to surveillance expands measures for surveillance quality beyond typical variables (e.g., data quality and analytic capabilities) to desired characteristics of a quality public health system. The question becomes: How can disease surveillance help public health services to be more population centered, equitable, proactive, health-promoting, risk-reducing, vigilant, transparent, effective, and efficient - the desired features of a quality public health system?

Any agency with a public health mission, or even a partial public health mission (e.g., tax-exempt hospitals), can use these measures to develop strategies that improve both the quality of the surveillance enterprise and public health systems, overall. At this time, input from stakeholders is needed to identify valid and feasible ways to measure how surveillance systems and practices advance public health quality. What exists now and where are the gaps?

\section{Methods}

Improving public health by applying quality measures to disease surveillance will require innovation and collaboration among stake- holders. This roundtable will begin a community dialogue to spark this process. The first goal will be to achieve a common focus by defining the nine quality aims identified in the HHS Consensus Statement. Attendees will draw from their experience to discuss how surveillance practice advances the public health aims and improves public health. We will also identify key research questions needed to provide evidence to inform decision-making.

\section{Results}

The roundtable will discuss how the current state of surveillance practice addresses each of the aims described in the Consensus Statement to create a snapshot of how surveillance contributes to public health quality and begin to articulate practical measures for assessing quality improvements. Sample questions to catalyze discussion include:

- How is surveillance used to identify and address health disparities and, thereby, make public health more equitable? What are the data sources? Are there targets? How can research and evaluation help to enhance this surveillance capability and direct action?

- How do we identify and address factors that inhibit quality improvement in surveillance? What are the gaps in knowledge, skills, systems, and resources?

-Where can standardization play a positive role in the evaluation of quality in public health surveillance?

- How can we leverage resources by aligning national, state, and local goals?

-What are the key research questions and the quality improvement projects that can be implemented using recognized models for improvement?

- How can syndromic surveillance, specifically, advance the priority aims?

The roundtable will conclude with a list of next steps to develop metrics that resonate with the business practices of public health at all levels.

\section{Keywords}

public health quality; metrics; framework

\section{References}

1. Honoré PA, Wright D, Berwick DM, Clancy CM, Lee P, Nowinski J, Koh HK. Creating a framework for getting quality into the public health system. Health Aff (Millwood). 2011 Apr;30(4):737-45.

\author{
*Peggy A. Honoré \\ E-mail: Peggy.Honore@hhs.gov
}

